\title{
LAS ESPECIES DE PSILOCYBE (FUNGI, BASIDIOMYCOTINA, AGARICALES) CONOCIDAS DE JALISCO (MEXICO) Y DESCRIPCION DE DOS NUEVAS PARA LA CIENCIA ${ }^{1}$
}

\author{
Gaston GuZMAn \\ Instituto de Ecología, A.C. \\ Apartado postal 63 \\ 91000 Xalapa, Veracruz
}

\section{RESUMEN}

Se discuten 11 especies de Psilocybe registradas del Estado de Jalisco de las 55 conocidas para México. La cita bibliográfica más antigua sobre Jalisco data de 1973. Psilocybe coprophila se registra por primera vez de dos localidades del Municipio de San Sebastián del Oeste. Se describen además dos especies nuevas: $P$. villarrealiae y $P$. laurae, ambas de la Sección Cordisporae y por ello con propiedades neurotrópicas. Con éstas son ocho las especies de Psilocybe conocidas con dichas propiedades de Jalisco.

\begin{abstract}
Eleven species of Psilocybe reported from the State of Jalisco of the 55 known from Mexico are discussed. The earliest bibliographic report from Jalisco dates from 1973. Psilocybe coprophila is reported for first time from two localites of the County of San Sebastián del Oeste. Moreover, $P$. villarrealiae and $P$. laurae are described as new species; they belong to the Section Cordisporae, and consequently present neurotropic properties. With these fungi, the neurotropic species of Psilocybe known from Jalisco are eight.
\end{abstract}

\section{INTRODUCCION}

La diversidad de los hongos en México es tan grande, que parece interminable su conocimiento (Guzmán, 1998). Después de la monografía mundial sobre Psilocybe (Guzmán, 1983), en donde se consideraron 144 especies en el género, 42 de ellas de México, se han descrito 34 especies adicionales (Guzmán, 1995) y posteriormente otras más. Se conocen actualmente de México 55 especies, cinco de ellas hace poco descritas o citadas de Jalisco y Veracruz (Guzmán et al., en prensa).

En una exploración botánica realizada en 1997 y organizada por el Instituto de Botánica de la Universidad de Guadalajara, a la región de San Sebastián del Oeste, en

\footnotetext{
1 Este trabajo forma parte del Proyecto Ordenamiento de la Vegetación, Flora y Micobiota de los Estados de Jalisco y Colima, de la Universidad de Guadalajara, bajo la dirección del Dr. J. Antonio Vázquez García.
} 
el Estado de Jalisco, el autor, en colaboración con la M. en C. Laura Guzmán-Dávalos, tuvo la oportunidad de recolectar ciertos hongos interesantes. En lo que respecta a los del género Psilocybe, unos especímenes resultaron ser nuevos registros para la entidad y otros pertenecen a nuevas especies, hechos que constituyen la base del presente trabajo.

\section{MATERIALES Y METODOS}

Se hizo una exhaustiva revisión bibliográfica de los hongos de Jalisco. Los especímenes fúngicos recolectados en la región de San Sebastián del Oeste, Jalisco, después de su herborización y registro, fueron estudiados al microscopio. Para esto último, se efectuaron cortes a navaja de los basidiomas y se montaron en $\mathrm{KOH}$ a $5 \%$, azul-algodón en lactofenol, rojo congo o solución de Melzer, según los requerimientos anatómicos. Los colores del basidioma se anotaron siguiendo la guía de Methuen (Kornerup y Wanscher, 1989).

\section{LAS ESPECIES DE PSILOCYBE EN JALISCO}

Son 11 las especies del género Psilocybe que se conocen del Estado de Jalisco, de las cuales Guzmán-Dávalos y Fragoza-Díaz (1994) consideraron nueve. En el Cuadro 1 se presentan tales especies, con las referencias bibliográficas correspondientes. Es interesante observar, que la referencia más antigua que registra especies de Psilocybe es de apenas hace 25 años: Guzmán y García-Saucedo (1973). Las especies de Psilocybe de Jalisco se adscriben a las siguientes secciones en el género: Coprophilae ( $P$. coprophila), Cordisporae ( $P$. caerulescens y $P$. cordispora), Cubensae ( $P$. cubensis y $P$. subcubensis), Merdariae ( $P$. pegleriana), Mexicanae ( $P$. galindoi, $P$. jaliscana y $P$. mexicana), Pratensae ( $P$. inquilina) y Zapotecorum $(P$. zapotecorum). Las especies de las secciones Cordisporae, Cubensae, Mexicanae y Zapotecorum tienen propiedades neurotrópicas, debido a sus características organolépticas y el manchado de color azul-verdoso de sus basidiomas, como lo ha observado el autor en el género (Guzmán, 1983). Dichas especies suman ocho, de las cuales $P$. cubensis y $P$. subcubensis son las más comunes, sobre todo la primera que procede de aproximadamente 10 localidades. Ambas crecen sobre estiércol vacuno o equino, en potreros de zonas tropicales y/o subtropicales. Por otra parte, $P$. coprophila se conoce de más de cinco localidades y se señala ahora por primera vez de otras dos más (véase más adelante). $P$. mexicana es común en el suelo de praderas subtropicales con pastos rasantes y con fuerte influencia de ganado equino. $P$. jaliscana y $P$. galindoi se conocen sobre humus en bosques mesófilos de montaña o de pino-encino muy húmedos. $P$. caerulescens, $P$. cordispora y $P$. zapotecorum crecen en los mismos bosques, pero directamente sobre suelo arcilloso recién removido y sin vegetación herbácea. $P$. inquilina es terrícola y solamente se conoce de un bosque subtropical xerófilo. Finalmente, $P$. pegleriana, que originalmente fue citada como $P$. merdaria (Fr.) Ricken de Jalisco, del Ingenio de Tamazula (Guzmán et al., 1977) y luego como P. pseudobullacea (Petch) Pegler (Guzmán, 1983; Guzmán et al., en prensa) de la misma localidad y del Estado de Veracruz, crece sobre bagazo de caña de azúcar. 
Guzmán: Las Especies de Psilocybe Conocidas de Jalisco y Descripción de Dos Nuevas

Cuadro 1. Las especies de Psilocybe conocidas de Jalisco.

* P. caerulescens Murrill (Guzmán et al., 1988)

P. coprophila (Bull.: Fr.) P. Kumm.

(Fierros y Guzmán-Dávalos, 1995; Guzmán, 1983; Guzmán y García-Saucedo, 1973; Guzmán et al., 1977; Guzmán-Dávalos y Nieves, 1984; Guzmán-Dávalos et al., 1983; Rodríguez et al., 1994; SantanaMichel et al., 1987; Téllez et al., 1988)

* P. cordispora R. Heim (Guzmán et al., 1988)

* P. cubensis (Earle) Singer (Guzmán, 1982, 1983; Guzmán et al., 1979, 1988; Guzmán-Dávalos, 1992; Guzmán-Dávalos et al., 1983; Guzmán-Dávalos y Nieves, 1984; Nieves, 1985; Rodríguez et al., 1994)

* P. galindoi Guzmán (Guzmán, 1978, 1983; Guzmán et al., 1988; Guzmán-Dávalos et al., 1983)

P. inquilina (Fr. : Fr.) Bres.

(Guzmán, 1995)

* P. jaliscana Guzmán (Guzmán et al., 1998)

* P. mexicana R. Heim (Guzmán, 1978, 1983; Guzmán et al., 1979, 1988; Guzmán-Dávalos et al., 1983; Guzmán-Dávalos y Nieves, 1984; Nieves, 1985)

$P$. pegleriana Guzmán

(= P. pseudobullacea (Petch) Pegler sensu Guzmán, 1983)

(Guzmán, 1983; Guzmán et al., 1977, 1979, 1998)

* P. subcubensis Guzmán

(Guzmán, 1978, 1983; Guzmán et al., 1979, 1988; Guzmán-Dávalos et al., 1983)

* P. zapotecorum R. Heim emend. Guzmán

(Guzmán et al., 1988; Guzmán-Dávalos y Nieves, 1984; González-Villarreal y Pérez de la Rosa, 1987)

* Especies con propiedades neurotrópicas

\section{NUEVAS LOCALIDADES PARA PSILOCYBE COPROPHILA}

Este hongo tiene amplia distribución por desarrollarse sobre diversos tipos de estiércol, principalmente equino y vacuno, en una amplia gama de variantes climáticas. Se ha citado de Jalisco (véase Cuadro 1) de numerosas localidades, pero no se conocía del Municipio de San Sebastián del Oeste, en donde se encontró en dos sitios, que se señalan aquí como nuevos registros para la especie: 1) camino San Sebastián del Oeste a La Bufa, 5 km de San Sebastián del Oeste, julio 12, 1997, Guzmán 32010 (IBUG, XAL) y 2) camino San Sebastián a La Bulera, La Mesa, julio 13, 1997, Guzmán 32041 (IBUG, XAL).

\section{DESCRIPCION DE ESPECIES NUEVAS}

Psilocybe villarrealiae Guzmán, sp. nov. Figs. 1-5.

Pileus 40-50 mm latus, convexus vel inaequaliter planus, vel subumbilicatus, laevis, hygrophanus, ochraceus vel albidus, subcaeruleus. Lamellae adnatae vel subsinuatae, 
fuscoviolaceae, ad margines albidae vel concolores. Stipes 50-60 x 4-7 mm, subsquamigerus, ochraceus vel rufobrunneus vel albidus, celeriter subcaeruleus vel fuscus. Caro albida vel lutescens, subcaerulea. Sporae (5.5-)6.5-7.5(-8)(-9) x (4.5-)5-6 $\mu \mathrm{m}$, frontaliter subrhombicae, lateraliter subellipsoideae. Pleurocystidia 12-29(-32) x (5-)6-7(-8) $\mu \mathrm{m}$, hyalina, subcommunia. Cheilocystidia (13-)(16-)19-25(-28) x (4-)5-6(-7.5) $\mu \mathrm{m}$, hyalina, communia. Pilepellis subgelatinosa. Hyphae fibulatae. Ad terra argillacea, in silva subtropica. Mexico, prope Jalisco, San Sebastián del Oeste, legit Guzmán 32009, holotypus XAL (isotypus IBUG).

Píleo de 40-50 mm de diámetro, convexo a irregularmente plano o algo umbilicado, con borde irregularmente ondulado o sublobulado, superficie lisa, con el margen finamente estriado a surcado en seco, intensamente higrófano, amarillo-pardusco brillante (4A4) a blanquecino-plateado opaco (3A2 o 22BI), con manchas irregulares azul-verdosas (25E5 o 25E7), a de color café obscuro (30F3) o con el margen verde obscuro (IE8-IF8); en seco es amarillento-pardusco opaco con tonos verdosos (4C6-4B5). Láminas adheridas en ángulo recto o un poco sinuadas, de color café-morado (9F5) o café-violáceo (13F5), con los bordes concoloros o blanquecinos. Estípite de 50-60 × 4-7 mm, central, uniforme en grosor o un poco bulboso, hueco, médula a veces rellena de micelio blanco; superficie amarillenta-pardusca clara (4A3), de color carne (5A2) o parda-rojiza (7A4), finamente cubierta de pequeñas escamas blanquecinas, con micelio algodonoso blanco en la base; toda la superficie queda blanquecina-plateada como el píleo; en seco se conserva de color café-rojizo obscuro (7E5-7F5) o café-negruzco (9F4); se mancha intensamente de verde moho (ID8) o se obscurece irregularmente de color café-rojizo (9F5). Velo ausente. Contexto blanquecino en el píleo y estípite o amarillento (4A4) en la base del pie, con olor y sabor farináceos, algo agrios; se mancha fácilmente de azul-verdoso (25E5).

Esporas de (5.5-)6.5-7.5(-8)(-9) x (4.5-)5-6 $\mu \mathrm{m}$, subrómbicas o algo ovoides en vista frontal o subelípticas en perfil, con pared semigruesa de hasta $1.5 \mu \mathrm{m}$ de grosor, lisa y de color café-amarillento, con poro germinal ancho en un extremo y un corto apículo en el opuesto. Basidios de 15-28 x 6-7 $\mu \mathrm{m}$, tetraspóricos, hialinos, ventricosos, a veces claviformes, generalmente con una constricción central. Se observaron basidiolos de 12.5-17 x 5-6.5 $\mu \mathrm{m}$ (Fig. 3c), con una aguda constricción central, imitando un tipo especial de pleurocistidios. Pleurocistidios de 12-29(-32) x (5-)6-7(-8) $\mu \mathrm{m}$, comunes, hialinos, de pared delgada, ventricoso-rostrados, con el ápice agudo y generalmente incrustado con material refringente; algunos pleurocistidios presentan cuello más o menos largo, de 2-3 $\mu$ m longitud (Fig. 4b) y otros, muy escasos, de hasta $7 \mu \mathrm{m}$ de longitud (Fig. 4c), en ambos casos con el cuello no mayor de $2 \mu \mathrm{m}$ de diámetro. Queilocistidios de (13-)(16-)19-25(-28) x (4-)5-6(-7.5) $\mu \mathrm{m}$, muy abundantes, hialinos, de pared delgada, ventricoso-rostrados (como los pleurocistidios) 0 subcilíndrico-sublageniformes, a veces submoniliformes, con un cuello de hasta $10 \mu \mathrm{m}$ de longitud, por $2 \mu \mathrm{m}$ de grosor y con el ápice ligeramente incrustado y refringente. Subhimenio subcelular, con elementos de 3-9 $\mu \mathrm{m}$ de diámetro, de pared delgada, hialinos y con incrustaciones de color café-amarillo en la pared. Trama regular, con hifas de (2-)4-18 $\mu \mathrm{m}$ de grosor, hialinas o amarillentas. Película del píleo subgelatinosa, con hifas de 1.5-2.5 $\mu \mathrm{m}$ de grosor, postradas, hialinas a amarillentas. Hipodermio con hifas de 4-6 $\mu \mathrm{m}$ de diámetro, de color café-amarillento. Fíbulas comunes. 

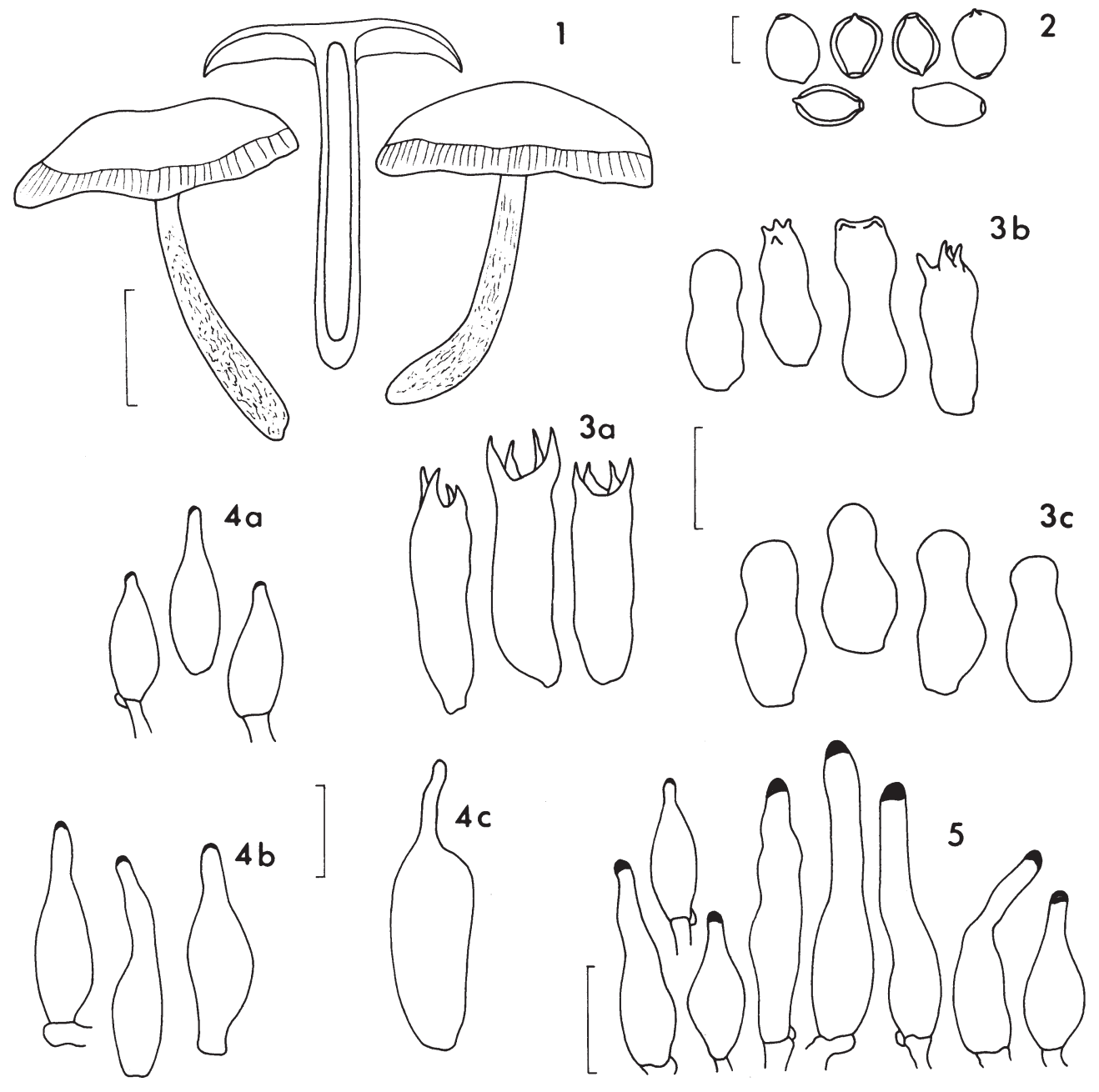

Figs. 1-5. Psilocybe villarrealiae. 1: basidiomas; 2 : esporas; 3a: basidios adultos; 3b: basidios jóvenes; 3c: basidiolos; 4a: pleurocistidios normales; 4b: pleurocistidios con cuello corto; 4c: pleurocistidio con cuello largo; 5: queilocistidios. Escala $20 \mathrm{~mm}$ en 1, $4 \mu \mathrm{m}$ en 2 y $9 \mu \mathrm{m}$ en 3-5. 
Holotipo: México, Jalisco, Municipio de San Sebastián del Oeste, camino San Sebastián del Oeste a La Bufa, 5 km de San Sebastián del Oeste, alt. 1300 m, 12.VII.1997, G. Guzmán 32009 (XAL) (isotipo en IBUG).

Hábitat y distribución: Gregario en suelo arcilloso y pedregoso, de color caféanaranjado-grisáceo, desprovisto de vegetación, a la orilla del camino, en un bosque mesófilo de montaña. Solamente conocido de la localidad típica. Convive con $P$. laurae, el cual fue encontrado a una distancia aproximada de $6 \mathrm{~m}$ (véase la discusión de $P$. laurae).

Discusión: Este hongo pertenece a la Sección Cordisporae Guzmán (Guzmán, 1983), por la forma de las esporas y el carácter cerulescente del basidioma. Es seguro que presente propiedades neurotrópicas, debido a que todas las especies de dicha sección las tienen. Por el color blanquecino del basidioma en la fase adulta y la esbeltez del mismo, semeja a $P$. caerulescens var. albida R. Heim (Heim et al., 1965-1966), hongo que fue considerado como sinónimo de $P$. caerulescens Murrill var. caerulescens por Guzmán (1983), por su amplia variabilidad en el color. $P$. villarrealiae se distingue de la especie de Murrill, por la ausencia de pleurocistidios en esta última. $P$. wrightii Guzmán, que solamente se conoce de la Provincia de Tucumán, Argentina, es otra especie relacionada, pero se diferencia por la forma de sus pleurocistidios, los cuales tienen ápice globoso, sin prolongación filamentosa y con cuello de 3-4 $\mu \mathrm{m}$ de grosor (Guzmán, 1983). Compárese con $P$. laurae con el cual es afín.

El nombre de la especie se dedica como homenaje, a la Profesora Luz María Villarreal de Puga, insigne maestra de la Universidad de Guadalajara y por los apoyos que siempre ha otorgado al autor en sus investigaciones.

Psilocybe laurae Guzmán, sp. nov. Figs. 6-11.

A Psilocybe villarrealiae Guzmán differt pleurocystidiis cum longo (10-115 $\mu \mathrm{m})$ collo, subannulo in stipite et papilla in pileo praesentibus. Ad terra argillacea, in silva subtropica. Mexico, prope Jalisco, San Sebastián del Oeste, legit Guzmán 32011, holotypus XAL (isotypus IBUG).

Píleo de 30-50 mm de diámetro, convexo a plano o ligeramente cóncavo, a veces umbonado con una aguda, pero corta papila, liso a finamente estriado en el margen, con el borde entero, lobulado o desgarrado; superficie higrófana, amarillenta-grisácea (4C4) irregularmente o amarillento-anaranjada (4A5-4A6); pasa rápidamente a blanquecinogrisácea (4A2 o 4B3); se mancha fácil e intensamente de verde-azuloso (26A4 o 25E8); en seco se presenta de color beige (4A5-4B5) con el margen negruzco. Láminas sinuadas, de color café-morado (9F5) o café-violáceo (13F5), con los bordes blanquecinos. Estípite de (20-)60-95 x 4-6(-8) mm, central, uniforme en grosor, a veces subbulboso, hueco, cubierto de mechas o escamas blancas o blanquecinas, principalmente hacia la base; amarilloanaranjado (4A3) a anaranjado-pardusco (5A7) o de color café-ladrillo (6C8); se mancha fácil e intensamente de verde-azuloso claro a obscuro (26E8 a 26F8); en seco es de color café-rojizo-negruzco (12F6-12F5) o casi negro. Velo aracnoide, blanco y mal definido en los estados adultos, pero forma un pseudoanillo escamoso en la parte apical del estípite. 
Guzmán: Las Especies de Psilocybe Conocidas de Jalisco y Descripción de Dos Nuevas

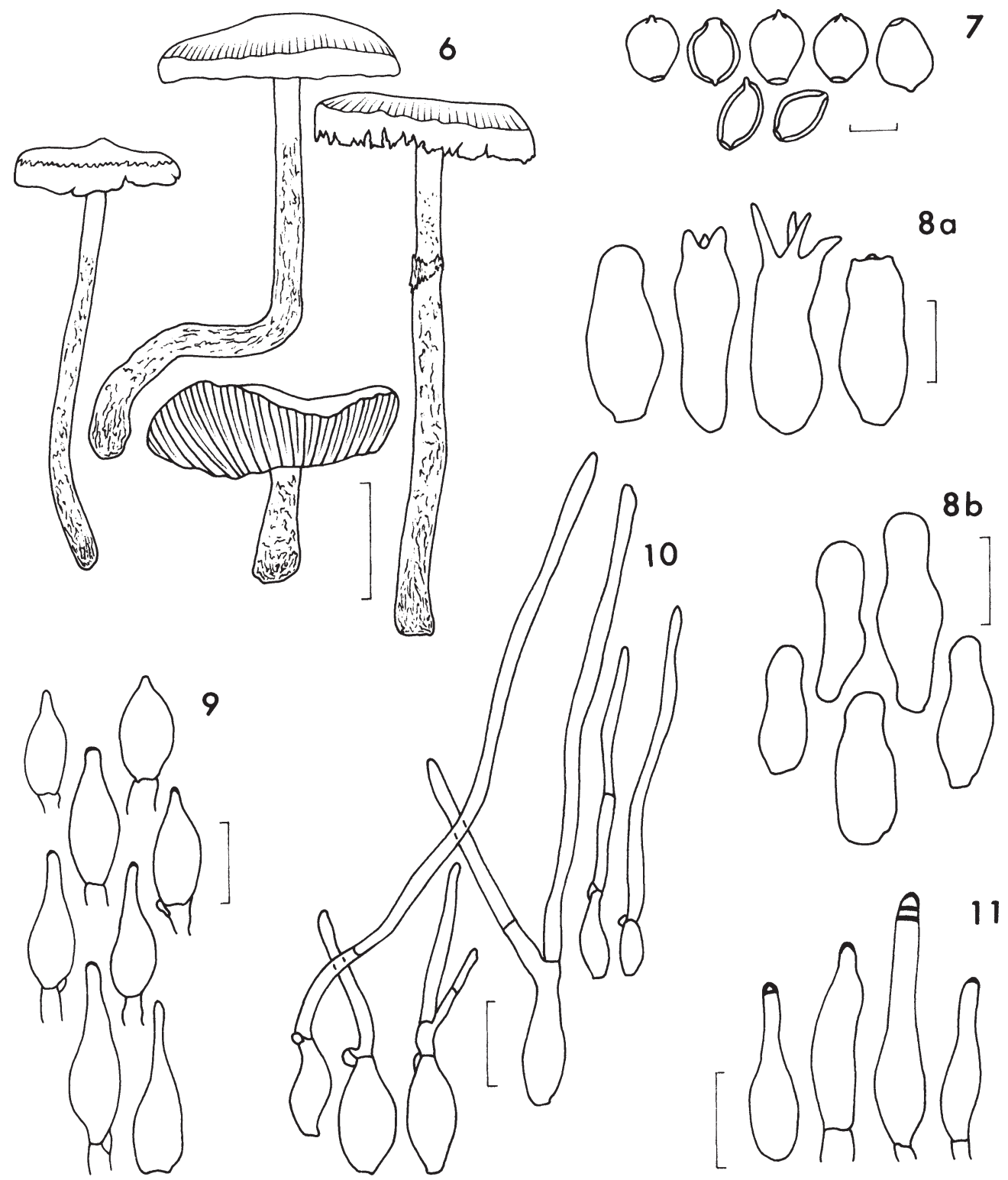

Figs. 6-11. Psilocybe laurae. 6: basidiomas; 7: esporas; 8a: basidios; 8b: basidiolos; 9: pleurocistidios del tipo 1; 10: pleurocistidios del tipo 2; 11: queilocistidios. Escala $20 \mathrm{~mm}$ en $6,4 \mu \mathrm{m}$ en 7 y $9 \mu \mathrm{m}$ en $7-11$. 
Contexto de consistencia fibroso-elástica y blanquecino en el píleo a subcartilaginoso y amarillento (4A3) en el estípite. Olor y sabor farináceo. Esporada (observada sobre el pseudoanillo) de color café-violáceo (11F8).

Esporas de 6.5-7.5(-8) x 5-6(-6.5) $\mu \mathrm{m}$, subrómbicas o ligeramente ovoides en vista frontal, subelipsoides de perfil, con pared semigruesa, de hasta $1.5 \mu \mathrm{m}$ de grosor, lisa y de color café-amarillento, con poro germinal ancho en un extremo y un apículo en otro. Basidios de 20-26 x 5-6(-7) $\mu \mathrm{m}$, tetraspóricos, hialinos, ventricosos o claviformes y con una constricción central; con basidiolos (Fig. 8b) de 15-21 x 6-8 $\mu \mathrm{m}$, ventricosos con una constricción media o casi apical, imitando pequeños pleurocistidios con cabeza globosa. Pleurocistidios de dos tipos, a saber: 1) de 12-18(-23) x (4-)5-6(-7) $\mu \mathrm{m}$ (Fig. 9), hialinos, de pared delgada, ventricoso-rostrados, con el ápice agudo y corto, o sublageniformes con cuello corto, no mayor de $4 \mu \mathrm{m}$ de longitud y con incrustaciones refringentes en el ápice y 2) de (22-)31-126 x (4.5-)6-7 um (Fig. 10), hialinos, de pared delgada, lageniformes, con un largo cuello de 10-115 × $2 \mu \mathrm{m}$, flexuoso, con una o dos tabicaciones y con una fíbula en la base del mismo; se presentan en forma abundante únicamente hacia el margen del píleo, en donde también están los del tipo 1. Queilocistidios de (13-)17-21(-24) x (5-)6-7(-8.5) $\mu \mathrm{m}$, abundantes, hialinos, de pared delgada, ventricoso-subcilíndricos o sublageniformes, con cuello corto a moderadamente largo, de $2 \mu \mathrm{m}$ de ancho y con incrustaciones refringentes en el ápice, a veces en forma de bandas. Subhimenio subcelular, con elementos de 3-8 $\mu \mathrm{m}$ de diámetro, de pared delgada, hialinos y con incrustaciones de color café-amarillento a ámbar. Trama regular, con hifas de 3-20 $\mu \mathrm{m}$ de diámetro, hialinas a amarillentas. Película del píleo subgelatinosa, con hifas de 1-2 $\mu \mathrm{m}$ de diámetro, hialinas. Hipodermio con hifas de 3-4 $\mu \mathrm{m}$ de diámetro, hialinas a amarillentas. Fíbulas comunes.

Holotipo: México, Jalisco, Municipio de San Sebastián del Oeste, camino San Sebastián del Oeste a La Bufa, 5 km de San Sebastián del Oeste, alt. 1300 m, 12.VII.1997, G. Guzmán 32011 (XAL) (isotipo en IBUG).

Hábitat y distribución: en el mismo hábitat y con la misma distribución que $P$. villarrealiae (véase éste).

Discusión: Psilocybe laurae es muy afín a $P$. villarrealiae, pero se diferencia por los pleurocistidios con el cuello largo, por la papila del píleo y el pseudoanillo del estípite, ausentes en aquella especie. Los pleurocistidios con las prolongaciones tan largas y cilíndricas, tabicadas y fibuladas en la base, son únicos en el género Psilocybe. Ninguna de las especies conocidas presenta tales estructuras (Guzmán, 1983; 1995), no así en otros géneros. Guzmán et al. (1997) observaron tales prolongaciones extremadamente largas y cilíndricas en los queilocistidios de Lentinula novaezelandiae (Stév.) Pegler, y Hilber (1982, p. 356, pl. 82) en los pleurocistidios de Pleurotus opuntiae (Dur. \& Lév.) Sacc.; en ambos casos con una fíbula en la base del filamento o a veces únicamente con tabiques en el filamento. Sin embargo, las observaciones de Guzmán et al. (1997) en la citada Lentinula novaezelandiae, fueron hechas únicamente en basidiomas obtenidas por cultivo, no así en el material silvestre del tipo, que carece de tales cistidios con las prolongaciones cilíndricas y parece que así ocurre con el Pleurotus opuntiae de Hilber. Será necesario efectuar estudios sobre el desarrollo del basidioma de Psilocybe laurae, a través de las cepas que 
se puedan obtener, y compararlos con los estudios que se hagan con cepas de $P$. villarrealiae, e incluso con las de $P$. wrightii discutido en $P$. villarrealiae.

La población de basidiomas estudiada de $P$. laurae consistió de cinco fructificaciones y crecía a una distancia aproximada de $6 \mathrm{~m}$ de la de $P$. villarrealiae formada por dos basidiomas. En todos los casos los basidiomas eran adultos.

El nombre de la especie está dedicado a la Maestra en Ciencias Laura GuzmánDávalos, Directora del Departamento de Botánica y Zoología de la Universidad de Guadalajara, en reconocimiento a su destacada dedicación a la micología.

\section{AGRADECIMIENTOS}

El autor agradece al Dr. J. Antonio Vázquez García, Director del Instituto de Botánica de la Universidad de Guadalajara, por haberlo invitado a formar parte del proyecto sobre la vegetación, flora y micobiota de Jalisco y Colima, lo que le dió la oportunidad de explorar la región de San Sebastián del Oeste. También reconoce a Fidel Tapia, del Instituto de Ecología, A.C. y a Laura Guzmán-Dávalos, de la Universidad de Guadalajara, por haber revisado críticamente el presente artículo. A L. Guzmán-Dávalos se le agradece asimismo su colaboración y apoyo en el trabajo de campo. Florencia Ramírez-Guillén del Instituto de Ecología, A.C., colaboró en las observaciones microscópicas. Juan Lara Carmona y María Eugenia Ramírez, del citado instituto, apoyaron al autor en diversas tareas de la presente contribución. A todas estas personas se les manifiestan las gracias.

\section{LITERATURA CITADA}

Fierros, M. de la L. y L. Guzmán-Dávalos. 1995. Inventario preliminar de los hongos macroscópicos de la Sierra de Quila, Jalisco, México. Bol. IBUG 3: 129-142.

González-Villarreal, L. M. y J. A. Pérez de la Rosa. 1987. Guía de la excursión botánica al Nevado de Colima, Jalisco. In: Guías de Excursiones Botánicas en México VIII. X Congreso Mexicano de Botánica. Guadalajara. pp. 101-139.

Guzmán, G. 1978. Further investigations of the Mexican hallucinogenic mushrooms with descriptions of new taxa and critical observations on additional taxa. Nova Hedwigia 29. Cramer. Lehre. pp. 625-664.

Guzmán, G. 1982. Nuevos datos sobre el género Psilocybe y descripción de una nueva especie en México. Bol. Soc. Mex. Mic. 17: 89-94.

Guzmán, G. 1983. The genus Psilocybe. Beih. Nova Hedwigia 74. Cramer, Vaduz. 439 pp. + 40 láms.

Guzmán, G. 1995. Supplement to the monograph of the genus Psilocybe. In: Petrini, O. y E. Horak (eds.), Taxonomic monographs of Agaricales. Bibl. Mycol. 159. Cramer. Berlín. pp. 91-141.

Guzmán, G. 1998. Inventorying the fungi in Mexico. Biodiversity and Conservation 7: 369-384.

Guzmán, G. y D. A. García-Saucedo. 1973. Macromicetos del Estado de Jalisco, I. Consideraciones generales y distribución de las especies conocidas. Bol. Soc. Mex. Mic. 7: 129-143.

Guzmán, G., L. Montoya y V. M. Bandala. 1988. Nuevos registros de los hongos alucinógenos del género Psilocybe en México y análisis de la distribución de las especies conocidas. Rev. Mex. Mic. 4: 255-265.

Guzmán, G., D. Salmones y F. Tapia. 1997. Lentinula boryana: morphological variations, taxonomic position, distribution and relationships with Lentinula edodes and related species. Rep. Tottori Mycol. Inst. 35: 1-28. 
Guzmán, G., F. Tapia, P. Navarro y F. Ramírez-Guillén. New species and new records of Psilocybe from Spain, U.S.A. and Mexico. Cript. Mycol. (en prensa).

Guzmán, G., L. Varela y J. Pérez-Ortiz. 1977. Las especies no alucinantes del género Psilocybe conocidas en México. Bol. Soc. Mex. Mic. 11: 23-33.

Guzmán, G., R. Vázquez y A. López. 1979. Distribución de las especies del género Psilocybe en México y descripción de una nueva especie. Bol. Soc. Mex. Mic. 13: 173-186.

Guzmán-Dávalos, L. 1992. Hongos macroscópicos de Jalisco: logros y perspectivas. Tiempos de Ciencia (Univ. de Guadalajara) 27: 55-59.

Guzmán-Dávalos, L. y G. Fragoza-Díaz. 1994. Los hongos registrados del Estado de Jalisco. Bol. IBUG 2: $109-160$.

Guzmán-Dávalos, L. y G. Nieves. 1984. Hongos del Estado de Jalisco, III. Bol. Inst. Bot. Univ. Guadalajara 5 (10): 21-34.

Guzmán-Dávalos, L., G. Nieves y G. Guzmán. 1983. Hongos del Estado de Jalisco, II. Especímenes depositados en el Herbario ENCB, 1a. parte. Bol. Soc. Mex. Mic. 18: 165-181.

Heim, R., R. Cailleux, R. G. Wasson y P. Thévernard. 1965-1966. Nouvelles investigations sur les champignons hallucinogènes. Arch. Mus. Nat. d'Hist. Nat. 9: 111-220, + 11 láms.

Hilber, O. 1982. Die gattung Pleurotus. Bibl. Mycol. 87. Cramer, Vaduz. 448 p. + 25 láms.

Kornerup, A. y J. H. Wanscher. 1989. Methuen handbook of colour. 3ra. ed., Methuen. Londres. 252 pp.

Nieves, G. 1985. Contribución al conocimiento de los macromicetos del bosque La Primavera, Zapopan, Jalisco. Tesis profesional. Universidad de Guadalajara. Guadalajara. 74 pp.

Rodríguez. O., M. Garza y L. Guzmán-Dávalos. 1994. Inventario preliminar de los hongos del volcán de Tequila, Estado de Jalisco. Rev. Mex. Mic. 10: 103-111.

Santana-Michel, F. J., H. J. Arreola-Nava y L. Scheinvar. 1987. Guía de excursión botánica a Lagos de Moreno y Ojuelos. In: Guías de Excursiones Botánicas en México VIII. X Congreso Mexicano de Botánica. Guadalajara. pp. 49-74.

Téllez, C., L. Guzmán-Dávalos y G. Guzmán. 1988. Contribución al conocimiento de los hongos de la Reserva de la Biósfera de la Sierra de Manantlán, Jalisco. Rev. Mex. Mic. 4: 123-130. 